\title{
Percutaneous liver biopsy: Reflections and refinements
}

\author{
Cameron N Ghent MD
}

$A_{p}^{1}$ lmost 50 years ago, Menghini (1) described the one-second percutaneous suction liver biopsy and the needle that bears his name and revolutionized the practice of hepatology. He described some refinements a few years later (2) but the technique has changed little since then. It is, therefore, timely to re-evaluate the place of this timehonoured diagnostic test, given the tremendous changes that have revolutionized hepatology since then.

Better diagnostic tests for many diseases have led to a decreased need for biopsy to make a diagnosis in many patients. The increasing resolution and sophistication of imaging modalities, such as ultrasound, computed tomography and magnetic resonance imaging, have decreased the need for obtaining liver tissue for histological examination in some patients, particularly those with focal

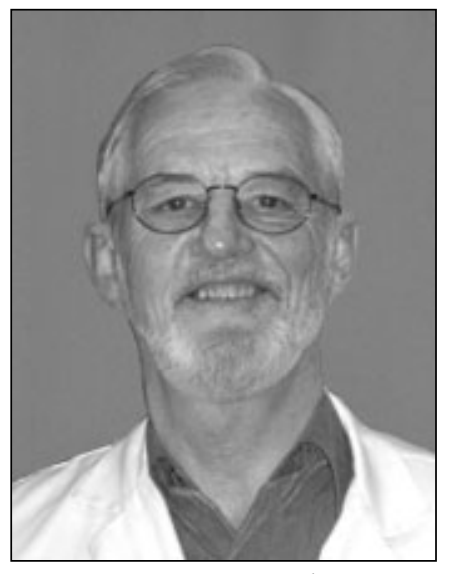

Dr Cameron N Ghent
The Canadian Association of Gastroenterology guidelines (11) are the most recent and do not differ substantially from earlier recommendations, but also do not meet the published arbitrary criteria for developing and using guidelines $(12,13)$.

This biased and personal review is based on over 30 years of performing and teaching the technique of percutaneous liver biopsy within academic health care centres and reviewing a large number of biopsy specimens with expert hepatopathologists. I will focus on some technical and some general aspects that are either neglected in other reviews or are commonly misunderstood by the neophyte gastroenterologists and hepatologists we attempt to teach. Alternative approaches to obtaining tissue such as transvenous biopsy, fine needle aspiration and operative or laparoscopic biopsy will only be mentioned when they are appropriate alternatives to routine transthoracic biopsy.

\section{INDICATIONS}

The indications listed in most reviews are of necessity very general and do not take into account many exceptions. A personalized list of common indications is shown in Table 1 . It is worthwhile reviewing some exceptions here.

Approximately $66 \%$ of the biopsies in my practice are for the staging of hepatitis $\mathrm{C}$, but it is worthwhile emphasizing that not everyone with chronic viral hepatitis needs a biopsy. If there are absolute contraindications to antiviral treatment, why do a 'pretreatment' biopsy? Likewise, someone with genotype 2 or 3 hepatitis $C$ has such a high probability of virological cure with treatment that routine pretreatment biopsy is not recommended (3). If risk factor analysis indicates that the hepatitis $\mathrm{C}$ has been present in the patient for fewer than 10 years, and in the absence of confounders such as alcohol abuse or obesity, the chances of severe fibrosis are very small and a trial of treatment without a biopsy for staging may be appropriate.

Conversely, the hepatitis $\mathrm{C}$ patient with clinical signs (spider nevi, palpable splenomegaly or ascites) or indirect laboratory evidence (thrombocytopenia or hypoalbuminemia) of cirrhosis is better served by performing an upper endoscopy to assess his or her risk for portal hypertensive bleeding than by

Department of Medicine, University of Western Ontario, London, Ontario

Correspondence: Dr Cameron N Ghent, University of Western Ontario, London, Ontario N6A 5A5. Telephone 519-642-3232,

fax 519-642-2545, e-mail drghent@on.aibn.com

Received for publication August 7, 2005. Accepted August 12, 2005 
TABLE 1

Common indications for percutaneous liver biopsy

\begin{tabular}{ll}
\hline Scenario & Comments \\
\hline Chronic hepatitis C & Genotype I, pretreatment staging \\
Chronic hepatitis B & Active disease, staging for choice of treatment \\
Suspected NASH & Confirmation, staging, elevated enzymes \\
Alcohol abuse & Staging and prognostication \\
PBC & Confirmation and staging, elective \\
PSC & Exclude overlap syndromes \\
Autoimmune hepatitis & For confirmation and staging, pretreatment \\
Elevated enzymes or ferritin & Very selective, usually low yield of serious disease \\
Liver graft dysfunction & Urgent need to exclude rejection \\
Fever of unknown origin & Culture a portion of specimen \\
\hline
\end{tabular}

NASH Nonalcoholic steatohepatitis; PBC Primary biliary cirrhosis; PSC Primary sclerosing cholangitis

doing a biopsy to confirm what the clinician already knows (ie, that the patient is cirrhotic). The same is true for the patient who is reported to have a frankly nodular liver contour on imaging studies - with the caveat that you are familiar with the reporting radiologist and know that he or she is reporting the nodularity from the images, not because the requisition includes the word cirrhosis. This anticipation phenomenon (seeing what you expect to see) plagues not only radiology reporting but that of pathologists and endoscopists, who are more likely to report the presence of varices if the patient is known to be cirrhotic, or to misdiagnose portal hypertensive gastropathy as chronic gastritis if there is no history of known liver disease.

Treatment decisions for chronic hepatitis B are increasingly being based on biochemical and virological measurements rather than on histology (3). Nevertheless, for those who prefer initial interferon treatment to treatment with nucleoside analogues, a pretreatment biopsy to exclude silent advanced cirrhosis is recommended.

Biopsy confirmation of suspected alcoholic liver disease is often appropriate, and has the added benefit of sending a subtle psychological signal to the patient that you take the problem seriously (emphasize the risks when obtaining consent). This psychotherapy can backfire if the very heavy drinker is found to have minimal disease and the clinician then loses strong ammunition in the battle to convince the patient to abstain.

Primary biliary cirrhosis is readily diagnosed without a biopsy in most patients (14), although confirmation of this diagnosis is cited as an indication for biopsy. Only in atypical presentations or when there is concern that there is overlap with autoimmune hepatitis is a diagnostic biopsy necessary.

However, many patients with primary biliary cirrhosis have sufficient concern about the stage of the disease that a biopsy for staging purposes may be appropriate. A few months after the diagnosis has been made, if the patient is constantly pondering the uncertainties of his or her disease, a biopsy for staging and reassurance may be appropriate - the indication for biopsy then becomes anxiety, not the need for confirmation of the diagnosis. It is noteworthy that the American Association for the Study of Liver Diseases practice guidelines for primary biliary cirrhosis recommend routine upper endoscopy in search of portal hypertensive changes at the time of diagnosis, but do not recommend universal liver biopsy for diagnosis (14).
Liver biopsy may be indicated in selected patients with suspected primary sclerosing cholangitis, but some form of cholangiography more readily diagnoses this disease. Besides the problem of considerable patchiness to the biliary obstruction in sclerosing cholangitis, leading to biopsy 'sampling error' histological features in biopsies of radiologically confirmed primary sclerosing cholangitis do not have prognostic significance (15).

The huge population of obese people with the metabolic syndrome dictates some selectivity in deciding to biopsy for confirmation of nonalcoholic fatty liver problems. This is an evolving recommendation - at this point in time I do not biopsy unless there are atypical features, elevated enzymes or physical features to suggest significant fibrosis or cirrhosis.

The asymptomatic patient who is found to have persistent elevation of serum enzymes or marked elevation of ferritin, usually in monitoring of some drug therapy, may be a candidate for a 'clear the air' biopsy if a thorough history, physical examination and selective laboratory testing do not result in a diagnosis. The expectation from a biopsy in this population is a low yield of a specific diagnosis for the problem, but a high yield of confirmation that the laboratory tests do not reflect serious silent liver disease.

The clear exception to internists, gastroenterologists and hepatologists doing biopsies is in the investigation of focal liver lesions. There is a general consensus that when such lesions need to be biopsied, ultrasound guidance and probably realtime ultrasound for the biopsy should be performed. The finding of a focal lesion on imaging in someone with known cirrhosis leads to a lot of debate about the wisdom of doing a fine needle aspiration to confirm the presence of hepatocellular carcinoma. If the serum alpha-fetoprotein is in the diagnostic range for hepatocellular carcinoma (greater than $1000 \mu \mathrm{g} / \mathrm{mL}$ ) or rapidly rising, or if there are no therapeutic implications, such as in the decompensated cirrhotic who is not a transplant candidate, a cytological diagnosis would seem unnecessary. There is a significant, although small ( $0 \%$ to $2.4 \%)$, risk of needle tract seeding in even fine needle aspiration of suspected hepatocellular carcinoma (16).

In the past, it has often sufficed to do fine needle aspirations of focal lesions if the probable diagnosis is some malignancy. However, with the increasingly narrow focus of oncology treatments to specific cell types of malignancy, it is increasingly necessary to get a more precise diagnosis than 'malignant cells present'; this need for precision in diagnosis in turn makes core biopsies, which can be studied for specific tumour markers, preferable to fine needle aspirates for the diagnosis of suspected hepatic malignancies. We have come full circle in the past few years from substituting a fine needle aspirate for a core biopsy in this situation, back to usually requesting a core biopsy.

\section{CONTRAINDICATIONS}

The classic contraindications to percutaneous liver biopsy outlined by Menghini (1) in his original description of the suction biopsy technique are still valid. There are, however, many gray areas. It is not clear at what level of thrombocytopenia the risk of bleeding complications increases, and the same is true for mild international normalized ratio (INR) elevations. As the need for a tissue diagnosis increases, the importance of some relative contraindications fades. Thus, in the liver transplant recipient with an urgent need to determine a cause of graft dysfunction, an inpatient percutaneous biopsy may be appropriate 
with a platelet count as low as $50 \times 10^{9} / \mathrm{L}$ to $60 \times 10^{9} / \mathrm{L}$, or an INR of 1.4 , but not both. The ready availability of coagulant support with fresh frozen plasma or platelet infusion in most transplant centres, and the alternative of transvenous biopsy, mean that contraindications can usually be circumvented if there is a very strong indication.

The presence of ascites is generally considered a contraindication to percutaneous biopsy. This is in part because with suction biopsies the suction may be lost in traversing the ascites, leading to the same risk and no sample, and in part because the ascites interposed between the chest wall and the liver may increase the risk of bleeding. The former problem can be readily overcome by a slight modification of the suction biopsy technique, simply by placing the needle through the ascites and slightly into the liver parenchyma before applying the syringe suction. The latter theoretical problem cannot be readily overcome but may not be important (see discussion below regarding postbiopsy positioning). This reviewer has performed several suction biopsies in patients with ascites of unknown cause, with no major complications.

Current guidelines recommend measurement of platelet count and INR within $24 \mathrm{~h}$ (American College of Gastroenterology [10]) or within one week (British Society of Gastroenterology [9]) before biopsy. While this is satisfying from an institutional and medical legal liability viewpoint, it creates logistical problems when patients travel long distances for outpatient biopsy, leads to unnecessary delays while waiting for the results and is simply not necessary. These measurements do not change rapidly in the course of chronic liver disease - even in patients stabilized on coumadin, the INR remains within a narrow range for intervals exceeding 14 weeks (17). For these reasons, our arbitrary practice is to insist on a laboratory report showing normal platelet count and INR performed within one month of the biopsy for stable outpatients. In acute disease situations, if previous tests have been significantly abnormal or when there are clinical changes in the patient's symptoms, an immediate prebiopsy measurement may be needed. Although some insist on routine measurement of partial thromboplastin time as well as INR, this reviewer could find no evidence that this was needed, recommended or necessary.

We routinely ask patients to forgo any use of acetylsalicylic acid, acetylsalicylic acid-containing products or nonsteroidal anti-inflammatory drugs for one week before elective outpatient biopsy; although there is no controlled trial evidence of decreased risk with this, doing the controlled trial to prove any benefit would be ethically problematic.

\section{TECHNICAL ASPECTS}

\section{Choice of needle}

There are two basic choices - a cutting needle or a suction needle. Since Menghini described the one-second suction technique, suction needles have been widely used, with significant modifications. Klatskin modified the Menghini needle to an internal bevel rather than an external bevel, with the theoretical advantage shown in Figure 1. Although fond of explaining his modification to fellows, Klatskin never published a record of it. The core of tissue obtained with an internal bevel needle is slightly larger than the bore of the needle, providing some radial compression of the specimen at the point of maximal insertion. This should provide a larger specimen for a given depth of insertion, because the specimen break is more likely to occur at or near the tip rather than some random point

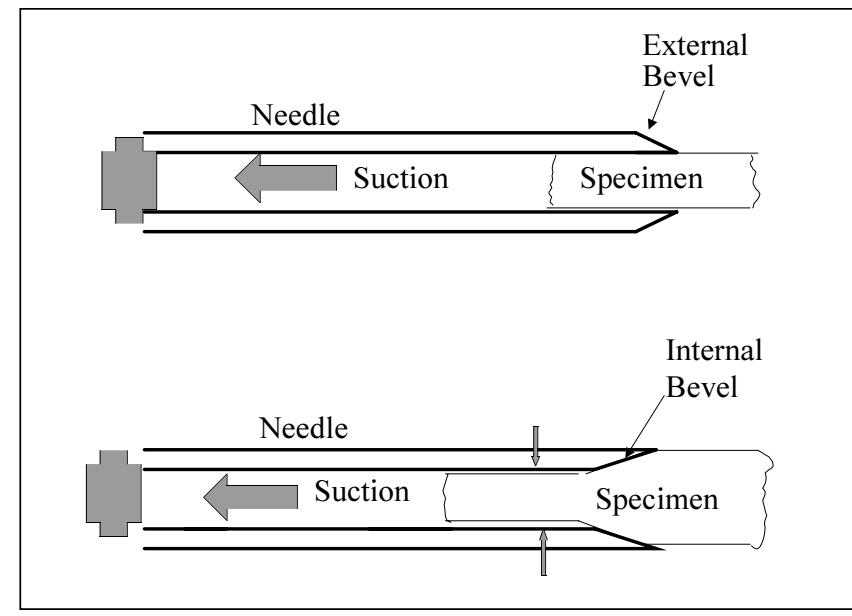

Figure 1) The rationale for use of an internal bevel needle (Klatsin or Jamshedi). Unlike an external bevel needle, internal bevel needles provide radial pressure on the specimen in the barrel of the needle (small arrows), theoretically increasing the chances the suction (large arrows) will cause tissue breakage at the point of maximum insertion, resulting in a larger specimen with the same risk to the patient

further up the needle bore. Jamshedi needles provide a similar theoretical advantage, the only major differences from the Klatskin needle are that they are disposable, intended for onetime use and are not equipped with the skin trochar, necessitating the use of a scalpel for skin puncture. (Klatskin needles need to be checked regularly for blunting of the bevelled tip and replaced when this is found, usually after approximately 40 uses).

Cutting needles and automated gun needles are used at many institutions and may provide an advantage in giving a more adequate and less fragmented specimen in severely cirrhotic patients (16). Patients known to have cirrhosis do not usually need a biopsy to determine the cause, which can more safely be determined by other means, so the use of cutting needles for this advantage implies their use for every biopsy where cirrhosis is a possible diagnosis - virtually everyone coming for biopsy. They require more double hand-eye coordination than this clinician could ever master; they seem unnecessarily complicated and are of necessity in the liver longer than a suction needle, theoretically increasing the risk of a capsular tear if the patient breathes. There are, however, conflicting data about the incidence of complications related to one type of needle or another (19).

\section{Patient preparation}

The British Society of Gastroenterology guidelines (9) recommend that all patients undergoing liver biopsy have some form of imaging of the liver within one month of the biopsy. This requirement either means that the patient make an extra trip to a radiology suite or has imaging done at the time of the biopsy. While there is a trend for gastroenterologists to let radiologists do both the imaging (usually ultrasound) and the biopsy, the value obtained for the extra cost of this has not been documented. Furthermore, most radiologists do not review their biopsies with pathologists regularly, a requirement for quality assurance that the biopsies are adequate. An alternative to doing biopsies in the ultrasound suite (either under real-time ultrasound guidance or after ultrasound marking of an appropriate skin site) is 
to educate gastroenterologists and hepatologists in the use of ultrasound to guide them in doing liver biopsies. Hepatologists will likely be using ultrasound, the stethoscope of the 21 st century, in routine office, clinic and bedside practice in any case within a few years, if or when territorial turf wars with radiologists are resolved and it becomes cost-effective to do so. There is a tendency for trainees to use ultrasound in place of percussion rather than as a supplement to percussion in finding the optimal site. This may be entirely appropriate with high-resolution scanners and personnel trained in ultrasound interpretation. At present, we use an antiquated, but adequate, ultrasound machine to check the position chosen for biopsy by percussion - one study indicated an infrequent need to change the site chosen by percussion if or when such ultrasound checks are routinely performed (20), but it does give some extra confidence that the position is appropriate. A similar assurance can be provided by using a $8.9 \mathrm{~cm} 22$ gauge spinal needle to infiltrate the local anesthetic, leaving this needle in place while the patient takes a breath to observe the needle movement as evidence of its transdiaphragmatic position; this manoeuvre, repeated as the 'sounding needle' is withdrawn, can also give a reasonable estimate of the depth to the diaphragm. This needle movement may not be observed in some early post-transplant patients whose right diaphragm may be immobile from a phrenic nerve apraxia because of the transplant surgery (21).

This reviewer was taught (in 1972) to have the patient lie on his or her left side, then to insert the needle downward into the liver. This position, it now seems, should increase the risk of a gas-filled loop of bowel interposing itself between the right diaphragm and the liver, increasing the chances of inadvertent bowel puncture. All guidelines now recommend positioning the patient supine with the right side at or hanging slightly over the side of the stretcher or bed, and inserting the needle horizontally (or in a slightly anterior and caudal plane) at a point below the level where dullness is first detected with progressive downward percussion in or near the midaxillary line. One manoeuvre which has not been emphasized but which greatly eases problems of finding adequate space between the ribs (and avoiding the neurovascular bundle running along the inferior margin of the rib) is to flex the patient to the left at the waist, thus separating the right ribs further. This can be very important in the obese subject whose ribs may only be palpable by this manoeuvre combined with stretching the right arm high over the patient's head, sometimes with an assistant retracting folds of adiposity.

Even with these positioning considerations, occasionally the intercostal space will only be found by trial and error with the local anesthetic needle.

\section{Further technical points}

Most suction biopsy needles are supplied with two unnecessary and quite useless pieces of equipment - the depth-control stop to be attached to the exterior of the needle to set the depth of penetration, and a specimen stop to be inserted into the hub of the needle to prevent the specimen from floating back into the saline-filled syringe. The depth-control stop can readily be replaced by a finger and thumb. In the Jamshedi needle set, the specimen stop is a separate pointed needle on a hub, attached to the syringe and fitted within the proximal end of the suction needle. This specimen stop is likewise not needed and may produce more crushing artifact to the specimen as it is impaled on a sharp tip than if it is allowed to float freely into the syringe. (This refinement would lend itself to a controlled trial if any pathologist is interested.) When the specimen stop is discarded, the operator is given the added immediate gratification of seeing the specimen in the syringe the second the needle is withdrawn, enabling him or her to immediately assure both patient and staff that the procedure is done and they can relax. The specimen can be transferred atraumatically from the syringe to the specimen container by removing the plunger from the syringe and pouring the saline-bathed specimen into an hourglass container, suctioning off the saline and then pouring formaldehyde onto the specimen for transfer to a specimen container.

\section{Postbiopsy care and complications}

No liver biopsy is free of risk. The period of observation required after outpatient biopsy varies among different institutions but certainly need not exceed $6 \mathrm{~h}$. It is my current practice to discharge patients who have had an uneventful single-pass biopsy after $3 \mathrm{~h}$ of observation, and require anyone needing an analgesic to stay under observation for at least $4 \mathrm{~h}$. The majority of major complications requiring hospitalization have been shown in prospective observational series to occur within $3 \mathrm{~h}$ of the biopsy (22), although later complications can ensue. The American College of Gastroenterology guidelines place further restrictions such as someone staying with the patient for $24 \mathrm{~h}$, and staying overnight within $30 \mathrm{~min}$ of the hospital. These limitations would greatly reduce the number of patients suitable for outpatient biopsy in many Canadian centres, and correspondingly increase the need for inpatient admissions specifically for biopsy. With the difficulties in accessing inpatient care within the current Canadian health care system, some degree of relaxation of these requirements would seem appropriate, though all patients should be advised about the need to seek help promptly if symptoms not present at discharge develop in the next few days.

Rates of complications vary in different case series and relate in part to operator experience, although the most experienced clinicians still will encounter complications. The risk of major complications is no higher than with many standard gastrointestinal procedures (lower than those associated with colonoscopic polypectomy [23]) but their abruptness and their unpredictability are probably major factors in the willingness of gastroenterologists to give up this procedure to ultrasonographers.

Standard lists of complications and their quoted rate ranges are shown in Table 2. A few of these deserve some comment. Intraoperative needle biopsy observations indicate that almost all patients have transient bleeding from the capsular puncture site. The figures in Table 2 refer to clinically significant bleeding resulting in pain, hypotension or the need for transfusions. The rate of many less serious complications depends on how diligently they are looked for. A typical example of this is the high incidence of arteriovenous fistulas (24). These are seldom significant but show up as new vascular lesions in imaging studies months or years after the biopsy, sometimes creating significant confusion as they are often thought to be vascular tumours.

As a guide in discussing the complications with patients to obtain consent, I quote them a one in 300 chance of suffering some adverse event that will require immediate hospitalization, a one in 1000 chance of requiring surgical intervention to deal with the complication and a one in 10 chance of having 
transient site or right shoulder pain severe enough to require some analgesic (and a nine in 10 chance of having a very boring morning lying on their right side on a hospital stretcher). The most common complication in my experience is actually a transient vasovagal reaction, manifested as sweating, nausea, hypotension and bradycardia, readily reversed with atropine administration.

It is standard practice to require the patient to lie on the right side for a variable period of time immediately following biopsy in an effort to provide some body weight tamponade to the capsular puncture site. This has never been subject to serious scrutiny and may not be beneficial at all - regardless of position, the diaphragm and liver are likely in the same relationship to each other and moving 12 to 20 times per minute with respiration. It is certainly not appropriate to deny biopsy to someone who, usually because of orthopedic problems, cannot lie on his or her right side.

In summary, percutaneous biopsy of the liver remains a valuable technique in the evaluation of many patients with both acute and chronic liver diseases, and should be taught to all trainees in gastroenterology programs. One of the greatest impediments to its more widespread appropriate use is the absence of specifically trained and interested hepatopathology

\section{REFERENCES}

1. Menghini G. One-second needle biopsy of the liver. Gastroenterology 1958;35:190-9.

2. Menghini G. One-second biopsy of the liver - problems of its clinical application. N Engl J Med 1970;283:582-5.

3. Sherman M, Bain V, Villeneuve JP, et al. The management of chronic viral hepatitis: A Canadian consensus conference 2004. Can J Gastroenterol 2004;18:715-28.

4. Shaffer EA. Nonalcoholic steatohepatitis: More than just being fat. Can J Gastroenterol 2002;16:318-21.

5. Tobkes AI, Nord HJ. Liver biopsy: Review of methodology and complications. Dig Dis Sci 1995;13:267-74.

6. Lebrec D. Various approaches to obtaining liver tissue - choosing the biopsy technique. J Hepatol 1996;25(Suppl 1):20-4.

7. Bravo AA, Sheth SG, Chopra S. Liver biopsy. N Engl J Med 2001;344:495-500.

8. Sheela H, Seela S, Caldwell C, Boyer JL, Jain D. Liver biopsy: Evolving role in the new millenium. J Clin Gastroenterol 2005;39:603-10.

9. Grant A, Neuberger J. Guidelines on the use of liver biopsy in clinical practice. British Society of Gastroenterology. Gut 1999;45(Suppl 4):1-11.

10. Jacobs WH, Goldberg SB. Statement on outpatient percutaneous liver biopsy. Dig Dis Sci 1989;34:322-3.

11. Buckley A, Petrunia D. Practice guidelines for liver biopsy. Canadian Association of Gastroenterology. Can J Gastroenterol 2000;14:481-2.

12. Shekelle PG, Woolf SH, Eccles M, Grimshaw J. Clinical guidelines: Developing guidelines. BMJ 1999;318:593-6.

13. Feder G, Eccles M, Grol R, Griffiths C, Grimshaw J. Clinical guidelines: Using clinical guidelines. BMJ 1999;318:728-30.
TABLE 2

Potential complications and the range in reported frequencies

\begin{tabular}{lc}
\hline Scenario & Reported frequency (\%) \\
\hline Pain at biopsy site or right shoulder & $0.056-22$ \\
Hemorrhage & \\
Intraperitoneal & $0.03-0.7$ \\
Intrahepatic or subcapsular & $0.059-23$ \\
Hemobilia & $0.059-0.2$ \\
Bile peritonitis & $0.03-0.22$ \\
Pneumothorax and/or pleural effusion & $0.08-0.28$ \\
Arteriovenous fistula & 5.4 \\
Anesthetic reaction & 0.029 \\
Biopsy of adjacent organs & $0.001-0.044$ \\
Death & $0.0083-0.03$ \\
\hline
\end{tabular}

experts in smaller centres. Referral of slides to a tertiary care pathologist may frequently be necessary for the community gastroenterologist to get an appreciation for the true contributions of histopathology to the decision-making process in caring for a patient with liver disease.

14. Heathcote JE. Management of primary biliary cirrhosis. The American Association for the Study of Liver Diseases practice guidelines. Hepatology 2000;31:1005-13.

15. Burak KW, Angulo P, Lindor KD. Is there a role for liver biopsy in primary sclerosing cholangitis? Am J Gastroenterol 2003;98:1155-8.

16. Abdalla EK, Vauthey JN. Technique and patient selection, not the needle, determine outcome of percutaneous intervention for hepatocellular carcinoma. Ann Surg Oncol 2004;11:240-1.

17. Lidstone V, Janes S, Stross P. INR: Intervals of measurement can safely extend to 14 weeks. Clin Lab Haematol 2000;22:291-3.

18. Goldner F. Comparison of the Menghini, Klatskin and Tru-Cut needles in diagnosing cirrhosis. J Clin Gastroenterol 1979;1:229-31.

19. Forssell PL, Bonkowsky HL, Anderson PB, Howell DA. Intrahepatic hematoma after aspiration liver biopsy. A prospective randomized trial using two different needles. Dig Dis Sci 1981;26:631-5.

20. Riley TR III. How often does ultrasound marking change the liver biopsy site? Am J Gastroenterol 1999;94:3320-2.

21. McAlister VC, Grant DR, Roy A, et al. Right phrenic nerve injury in orthotopic liver trasnsplantation. Transplantation 1993;55:826-30.

22. Knauer MC. Percutaneous biopsy of the liver as a procedure for outpatients. Gastroenterology 1978;74:101-2.

23. Gatto N, Frucht H, Sundararajan V, Jacobson JS, Grann VR, Neugut AI. Risk of perforation after colonoscopy and sigmoidoscopy: A population based study. J Natl Cancer Inst 2003;95:230-6.

24. Okuda K, Musha H, Nakajima Y, et al. Frequency of intrahepatic arteriovenous fistula as a sequela to percutaneous needle puncture of the liver. Gastroenterology 1978;74:1204-7. 


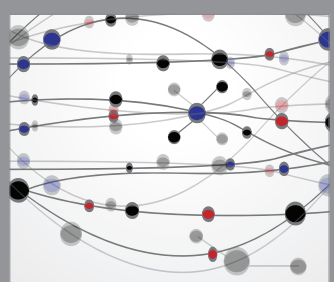

The Scientific World Journal
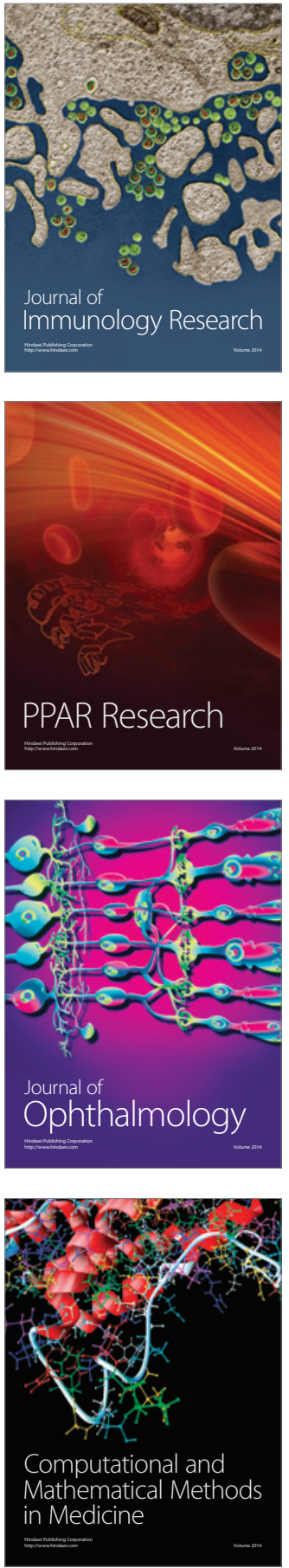

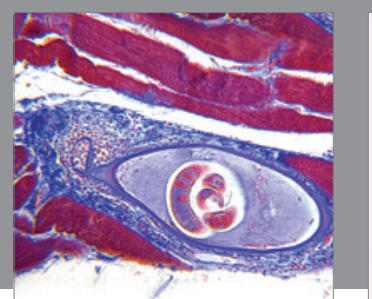

Gastroenterology Research and Practice

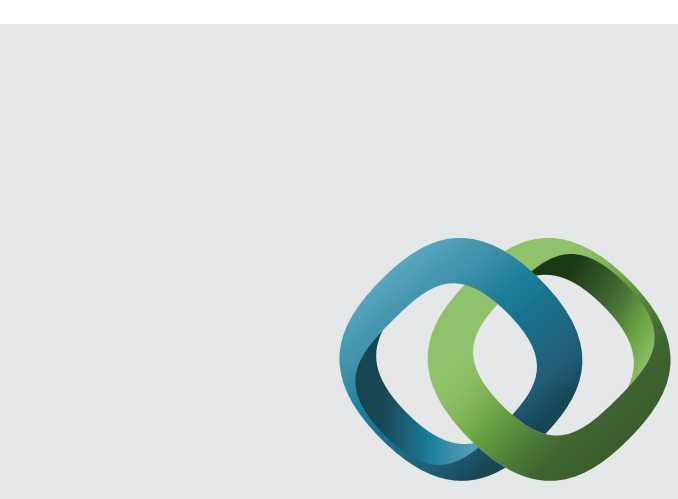

\section{Hindawi}

Submit your manuscripts at

http://www.hindawi.com
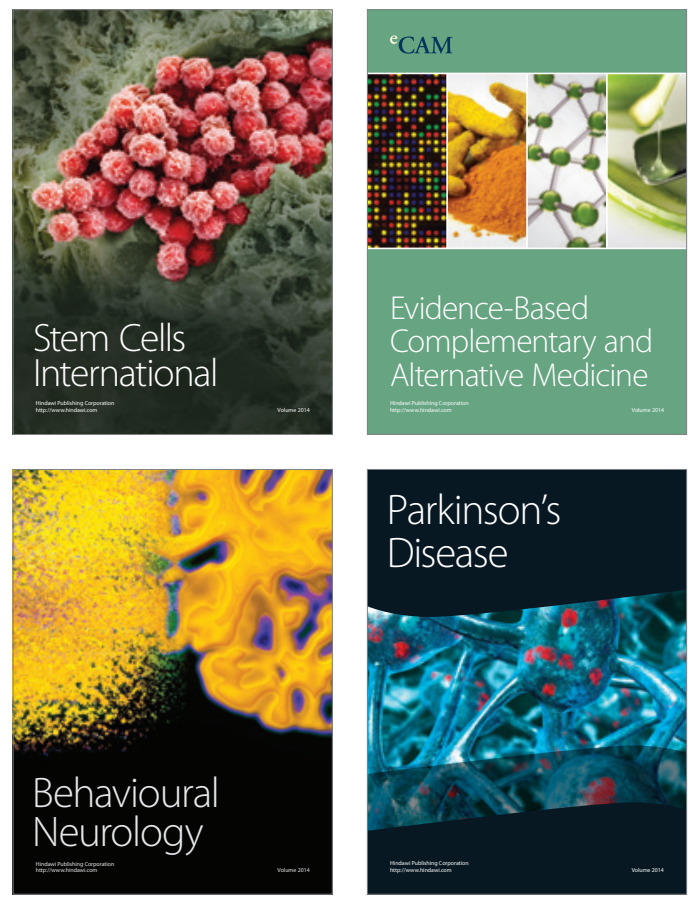
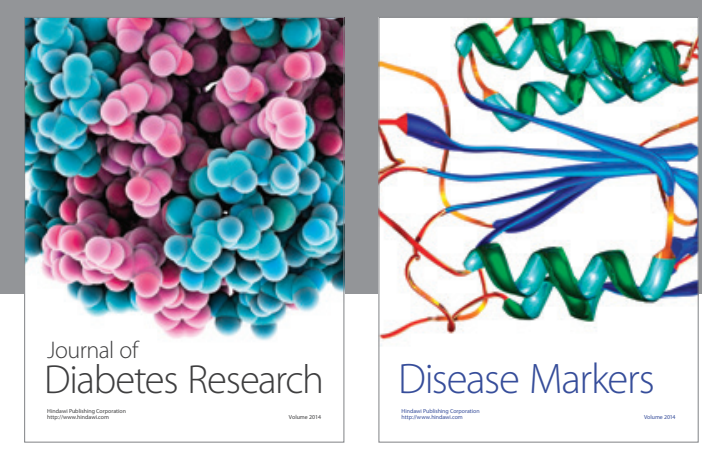

Disease Markers
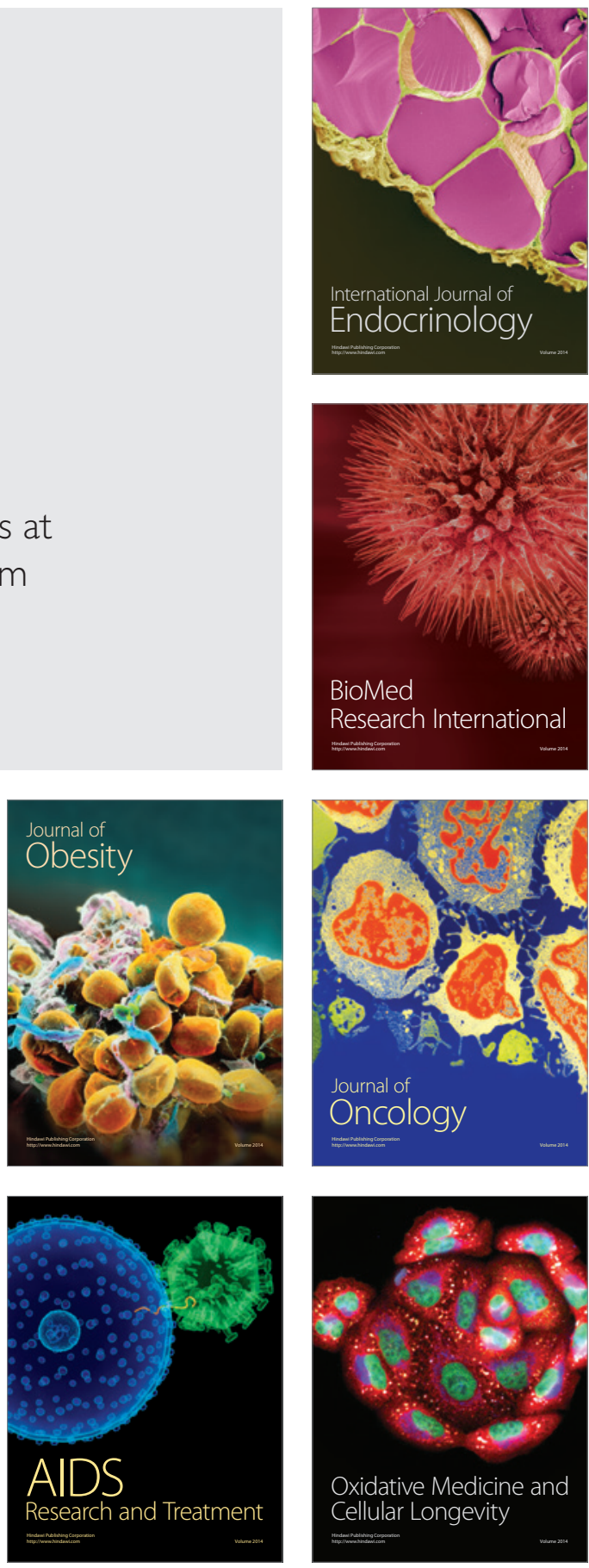